\title{
Successful treatment of aconitine induced life threatening ventricular tachyarrhythmia with amiodarone
}

\author{
D-F Yeih, F-T Chiang, S K S Huang
}

\begin{abstract}
With the increasing trend of cross mixing of populations, aconitine induced poisoning and its related arrhythmias may be more frequently encountered worldwide. However, the clinical experience is often too limited to draw any conclusion on the optimal treatment for tachycardia induced by aconitine intoxication. The clinical presentation, serial electrocardiographic changes, and responses to antiarrhythmic agents are reported in a patient with aconitine induced life threatening ventricular tachyarrhythmia. Amiodarone was effective in suppressing polymorphic ventricular tachycardia, which might provide an example of successful pharmacological intervention in aconitine induced ventricular tachyarrhythmia.

(Heart 2000;84:e8)
\end{abstract}

Keywords: aconitine; herbal medicine; life threatening ventricular tachyarrhythmia; amiodarone

Accepted 2 June 2000
Herbal drugs are widely used for treatment of many common medical problems throughout the world. They are thought to be natural, harmless, and to have minimal side effects. However, herbal drugs can sometimes lead to fatal poisoning. ${ }^{1}$ Among them, dried rootstocks of Aconitum plants are most notorious for cardiac toxicities. ${ }^{2}$ We report a case in which a person developed life threatening polymorphic and bidirectional ventricular tachyarrhythmias after ingestion of a bowl of herbal decoction, prepared from the rootstocks of Aconitum. Intravenous amiodarone infusion effectively suppressed the ventricular tachyarrhythmia which was refractory to intravenous lidocaine.

\section{Case report}

A 61 year old previously healthy man was admitted with sudden onset of severe palpitation, chest discomfort, and perioral paraesthesia. He had suffered from low back pain and arthralgia for the past three months and received some prescribed analgesics for pain relief but in vain. He therefore took some Chinese herbal medicine for his intractable pain without prescription. One hour before admission, he ingested a bowl of a herbal decoction, prepared from boiling a combination of herbs including the processed rootstocks of Aconitum. On examination, the patient remained alert during tachycardia despite an acute ill looking appearance with cold sweating. His blood pressure was $90 / 65 \mathrm{~mm} \mathrm{Hg}$ and pulse rate was 108 per minute. The 12 lead ECG revealed intermittent polymorphic ventricular tachycardia and occasional bidirectional alternation in QRS configurations (fig 1). Serum creatine kinase and electrolytes were within normal limits. Lidocaine $100 \mathrm{mg}$ intravenous bolus was given, followed by continuous infusion at a rate of $2 \mathrm{mg} / \mathrm{min}$ for the ventricular tachyarrhythmia. Unfortunately, there were still episodes of polymorphic ventricular tachycardia with haemodynamic compromise. Therefore, amiodarone $150 \mathrm{mg}$ was slowly infused over 15 minutes, followed by continuous infusion of $1300 \mathrm{mg}$ for the following 36 hours. The ventricular tachyarrhythmia stopped and the patient regained normal sinus rhythm one hour after amiodarone treatment (fig 2). A total of $1450 \mathrm{mg}$ of amiodarone was given without further administration of oral amiodarone. Predischarge evaluations showed normal ECG, echocardiography, and coronary angiography.

\section{Discussion}

Throughout the world, aconites have long been taken for many medical problems, including

Figure 2 Sinus bradycardia after administration of intravenous amiodarone. 
arthralgia, neuralgia, and some cardiac complaints. ${ }^{2}$ In Chinese medicine, the Aconitum rootstocks are called "Chuan-wu" and are processed by soaking or boiling them in water, which leads to hydrolysis of aconite alkaloids into less toxic derivatives such as aconines. ${ }^{2}$ Toxicity of aconites has also been recognised. Severe poisoning has been reported after ingestion of as little as $0.2 \mathrm{mg}$ pure aconitine, or consumption of decoctions prepared from prescriptions containing $6 \mathrm{~g}$ of cured Aconitum rootstocks. ${ }^{2}$ Aconite alkaloids contain variable amounts of highly toxic compounds, including aconitine, mesaconitine, and hypaconitine. The amount and composition of Aconitum alkaloids are the main factors determining the severity of intoxication and vary greatly with different species, time of harvesting, and the method of processing. ${ }^{2}$ Although the curing process reduces the toxicity of the aconites, patients may not follow the instructions precisely and the variable amounts of active ingredients within the root make the effect of this process unpredictable. ${ }^{3}$ The main causes of death are cardiovascular collapse caused by ventricular tachyarrhythmia. ${ }^{4}$

Aconitine and its related alkaloids can bind with high affinity to the open state of sodium channels, thus causing a persistent activation of sodium channels by blocking its inactivation. Enhancement of transmembranous inward current during the plateau phase of the action potential prolongs repolarisation of conduction fibres and induces afterdepolarisation with triggered activity, resulting in supraventricular or ventricular tachyarrhythmias. ${ }^{56}$ In addition, activation of muscarinic receptors also plays an important role in bradyarrhythmia and hypotension. Widespread excitation of cellular membranes may also depress the neuromuscular transmission, resulting in paraesthesia, numbness in the mouth and limbs, and progressive muscular weakness. ${ }^{4}$

The management of lethal ventricular tachyarrhythmia associated with Aconitum poisoning presents a serious therapeutic challenge. The clinical experience is too limited to draw any conclusion on the optimal treatment for tachycardia induced by aconitine intoxication. There is no specific antidote and the current treatment is essentially supportive. Data from animal studies on pharmacological intervention for aconitine induced tachyarrhythmia are limited and controversial. Winslow and colleagues showed that atrial fibrillation induced by direct aconitine application to cat atria could be suppressed by amiodarone. ${ }^{7}$ However, some investigators showed that amiodarone was ineffective in suppressing aconitine induced ventricular arrhythmia. ${ }^{8}$ In humans, to our knowledge, there has been only one large study reported by Tai and colleagues. ${ }^{6}$ They showed that nine of 23 patients with aconitine induced ventricular tachyarrhythmia were successfully controlled by antiarrhythmic agents. Five of these patients were treated with intravenous amiodarone. Lidocaine was ineffective in all patients. However, the exact dosage of amiodarone and temporal response was not reported. We demonstrated that amiodarone could be used effectively and safely to treat aconitine induced ventricular tachyarrhythmia after failure of intravenous lidocaine. The mechanism by which amiodarone exerts a beneficial effect on aconitine induced ventricular tachyarrhythmia is unclear. Amiodarone can prolong myocardial action potential, which may potentiate its inhibitory effect on use dependence sodium channels. In addition, amiodarone exhibits inhibition of calcium channels. These pharmacological effects can suppress triggered activity arising from early afterdepolarisation, which is suggested to be the underlying cause of aconitine induced ventricular tachyarrhythmia. ${ }^{9}$ Finally, amiodarone decreases QT dispersion in myocardium and then reduces the vulnerability of sustained ventricular tachycardia. ${ }^{10}$ This may also play an important role in suppressing aconitine induced ventricular tachyarrhythmia.

With the increasing trend of cross mixing of populations, aconitine induced poisoning and its related arrhythmias may be more frequently encountered world wide. A high index of suspicion is necessary for those who are presenting with hypotension and malignant cardiac arrhythmias. Amiodarone effectively suppressed the polymorphic ventricular tachycardias refractory to intravenous lidocaine. This may provide an example of successful pharmacological intervention in aconitine induced ventricular tachyarrhythmias.

1 De Smet PAGM. Toxicological outlook on the quality assurance of herbal remedies. In De Smet PAGM, Keller K, Hansel R, Chandler RF, eds. Adverse effects of herbal drugs, Hansel R, Chandler RF, eds. Adverse effects of
volume 1. Berlin: Springer-Verlag, 1992,1-72.

2 Chan TYK, Critchley JAJH. Usage and adverse effects of Chinese herbal medicines. Hum Exp Toxicol 1996;15:5-12. 3 Tomlinson B, Chan TYK, Chan JCN, et al. Herb-induced aconitine poisoning. Lancet 1993;341:370-1.

4 Chan TYK, Chan JCN, Tomlinson B, et al. Chinese herbal medicines revisited: a Hong Kong perspective. Lancet 1993;342:1532-4

5 Sawanobori T, Hirano Y, Hiraoka M. Aconitine-induced delayed afterdepolarization in frog atrium and guinea pig papillary muscles in the presence of low concentrations of papillary muscles in the presence

6 Tai YT, But PPH, Young K, et al. Cardiotoxicity after accidental herb-induced aconite poisoning. Lancet 1992;340: 1254-6.

7 Winslow E. Hemodynamic and arrhythmogenic effects of aconitine applied to the left atria of anesthetized cats: effects of amiodarone and atropine. $\mathcal{F}$ Cardiovasc Pharmacol 1981;3:87-100.

8 Gutierrez B, Vilumara A, Farre AJ. Inhibition of aconitineinduced mortality in the conscious rat: a screening test for anti-arrhythmic drugs. Methods Find Exp Clin Pharmacol 1987;9:307-10.

9 Tai YT, Lau CP, But PPH, et al. Bidirectional tachycardia induced by herbal aconitine poisoning. PACE 1992;15: $831-9$.

10 Zipes DP. Management of cardiac arrhythmia: pharmacological, electrical, and surgical techniques. In: Braunwald E, ed. Heart disease. Philadelphia: Saunders, 1997:593-639. 